\section{Mukosales Melanom: Kombination geprüft}

\author{
Mit einer gepoolten Datenanalyse wurde erstmals die Sicherheit und \\ Wirksamkeit von Nivolumab als Monosubstanz und in Kombination mit \\ Ipilimumab beim seltenen, hochaggressiven Schleimhautmelanom untersucht.
}

$\mathrm{M}$ ukosale Melanome machen in der weißen Bevölkerung nur etwa $2 \%$ aller Hautkrebstypen aus, in chinesischen Populationen hingegen bis zu $23 \%$. Diese hochaggressive Subtypen entziehen sich weitgehend den traditionellen Therapieansätzen. Auch gestaltet sich die komplette chirurgische Resektion aufgrund der anatomischen Lokalisation der Tumoren schwierig. Daten zur Aktivität von Ipilimumab und gegen PD-1 gerichtete Agenzien beruhen auf Studien mit geringer Fallzahl, retrospektiven Analysen und Einzelfallstudien. Um das Wissen zum möglichen Benefit einer Anti-PD1-Therapie des mukosalen Melanoms zu mehren, führten die Autoren eine gepoolte Datenanalyse zu Patienten mit diesem
Subtyp durch, die im Rahmen klinischer Untersuchungen entweder Nivolumab als Monoagens oder in Kombination mit Ipilimumab erhalten hatten.

Für ihre Analyse fassten sie die Daten von 889 Patienten zusammen, die ausschließlich den PD-1-Rezeptorblocker erhalten hatten, davon $10 \%$ mit einem mukosalen und $75 \%$ mit einem kutanen Melanom. Zudem poolten sie die Daten von 35 Patienten mit einem mukosalen und 326 mit einem kutanen Melanom, die mit der Kombination Nivolumab/ Ipilimumab behandelt wurden. Für die Gruppe unter Monotherapie und mit dem mukosalen Subtyp ermittelten die Autoren ein medianes progressionsfreies Überleben (PFS) von drei Monaten und objektives Ansprechen (ORR) von 23,3\%. Die Zahlen für Patienten mit einem kutanen Melanom: 6,2 Monate und $40,9 \%$. Vorteile in diesen beiden Parametern bescherte die Kombinationstherapie: 5,9 Monate und 11,7 Monate medianes PFS (mukosales vs. kutanes Melanom) und entsprechend ORR von $37,1 \%$ und $60,4 \%$. Die Rate an therapieassoziierten unerwünschten Ereignissen vom Grad 3 oder 4 war in der Kombinationsgruppe erhöht.

Fazit: Nach den Ergebnissen dieser umfangreichsten Datenanalyse zum Einsatz einer gegen PD-1 gerichteten Therapie scheint die Kombination Nivolumimab/ Ipilimumab der Monotherapie mit einem dieser Substanzen überlegen $\mathrm{zu}$ sein bei vergleichbarem Sicherheitsprofil. Wolfgang Zimmermann

D'Angelo SP et al. Efficacy and safety of nivolumab alone or in combination with ipilimumab in patients with mucosal melanoma: a pooled analysis. J Clin Oncol 2017; 35: 226-35

\title{
Wie riskant sind Gerinnungshemmer bei Operationen an der Haut?
}

Deutsche Hautchirurgen haben prospektiv erfasst, wie häufig bei Patienten mit antithrombotischer Therapie nach Hautoperationen Blutungen auftreten. Vom Bridging einer Phenprocoumontherapie raten sie ausdrücklich ab.

U $\mathrm{m}$ die Blutungsrisiken bei hautchirurgischen Patienten unter Antikoagulanzien oder Thrombozytenaggregationshemmern besser einschätzen zu können, haben deutsche Dermatochirurgen eine große prospektive Untersuchung begonnen. An der Studie waren 9.154 konsekutive Patienten aus neun hautchirurgischen Zentren beteiligt. Die meisten Eingriffe galten Basalzellkarzinomen, malignen Melanomen und Plattenepithelkarzinomen.

Insgesamt wurde bei 7,14\% der Patienten eine postoperative Blutung registriert, wobei die Notwendigkeit eines Verbandswechsels bereits als Blutung gewertet wurde. Schwere Blutungen, die ein ärztliches Eingreifen erforderten, ereigneten sich bei 83 Patienten, einem Anteil von $0,91 \%$. Sie konnten meistens mit dem Elektrokauter gestillt werden, nur bei 15 Patienten, also $0,16 \%$ aller Patienten, verliefen sie ungünstiger, mit Revision ( $\mathrm{n}=9)$, Transfusion $(\mathrm{n}=2)$ oder Nekrose $(\mathrm{n}=4)$.

Von den Patienten, die zum Zeitpunkt der OP ein oder zwei Gerinnungshemmer erhielten ( $\mathrm{n}=330$ bzw. 424), erlitten $1,50 \%$ und $1,18 \%$ eine schwere Blutung. Von den Patienten ohne antithrombotische Therapie waren es nur $0,53 \%(n=27)$. Bis auf eine Ausnahme handelte es sich dabei um Patienten, die nie gerinnungshemmend behandelt worden waren. Von den 325 Patienten, die eine antithrombotische Therapie unterbrochen hatten, entwickelte nur ein einziger - ein vormaliger ASS-Anwender - eine schwere Blutung. Bei fortgesetzter Behandlung mit nur einem Ge- rinnungshemmer ereigneten sich die meisten Blutungskomplikationen mit Clopidogrel $(2,86 \%, 3 / 105)$, gefolgt von Phenprocoumon $(2,28 \%, 15 / 657)$, ASS $(1,42 \%, 18 / 1267)$ und niedermolekularen Heparinen (NMH: 0,6\%, 6/1.014). Die Raten unter zwei Antithrombotika variierten zwischen $3,57 \%$ (ASS plus Clopidogrel) und 1,32\% (ASS plus NMH). Die mit 9,26\% höchste Blutungsquote überhaupt hatten Patienten, die Phenprocoumon vorübergehend durch ein $\mathrm{NMH}$ ersetzt hatten.

Fazit: Schwere Blutungen bei Hauteingriffen sind insgesamt selten; unter gerinnungshemmenden Wirkstoffen ist das Risiko generell leicht erhöht. Trotzdem raten die Autoren, eine Therapie mit Plättchenhemmern nicht zu unterbrechen. Auch Vitamin-K-Antagonisten können in der Regel weitergegeben werden. Auf die häufige Praxis der „Überbrückung“ mit Heparin sollte verzichtet werden.

Dr. Beate Schumacher

Koenen $\mathrm{W}$ et al. Prospective multicentre cohort study on 9154 surgical procedures to assess the risk of postoperative bleeding - a DESSI study. J Eur Acad Dermatol Venereol 2017; 31: 724-31 